\title{
Reliability, Resiliency, and Vulnerability Criteria For Water Resource System Performance Evaluation
}

\author{
TsUYoshi Hashimoto ${ }^{1}$ \\ International Institute for Applied Systems Analysis, Laxenburg, Austria
}

Jery R. Stedinger and Daniel P. Loucks

School of Civil and Environmental Engineering, Cornell University, Ithaca, New York 14853

\begin{abstract}
Three criteria for evaluating the possible performance of water resource systems are discussed. These measures describe how likely a system is to fail (reliability), how quickly it recovers from failure (resiliency), and how severe the consequences of failure may be (vulnerability). These criteria can be used to assist in the evaluation and selection of alternative design and operating policies for a wide variety of water resource projects. The performance of a water supply reservoir with a variety of operating policies illustrates their use.
\end{abstract}

\section{INTRODUCTION}

The ability of existing and proposed water resource systems to operate satisfactorily under the wide range of possible future demands and hydrologic conditions is an important system characteristic. The likely performance of water resource systems is often described by the mean and variance of benefits, pollutant concentrations, or some operating variable. This paper develops additional performance criteria that capture particular aspects of possible system performance which are especially important during periods of drought, peak demands, or extreme weather. The proposed criteria are called reliability, resiliency, and vulnerability. These performance measures should be useful in the selection of water resource system capacities, configurations, operating policies, and targets.

Bayesian methods are one natural and rigorous way of dealing with the uncertainty which arises in many planning studies. Davis et al. [1972] and Benjamin and Cornell [1970] review the basic methodology. When Bayesian analysis is combined with multiattribute utility theory [Keeney and Raiffa, 1976], the analysis can incorporate the variability in system performance and uncertainty in planning parameters with a single decision maker's attitudes toward risk. Examples of the use of multiattribute utility theory in water resources planning are given by Keeney and Wood [1977], Goicoechea et al. [1979] and Krzysztofowicz and Duckstein [1979].

Unfortunately, there are several drawbacks to this methodology. In particular the method requires the development of a utility function which incorporates a decision maker's or society's tradeoffs between competing system attributes and also their attitudes toward risk. Not only is such a function very difficult to construct for a single identified 'decision maker,' but such a function will probably not reflect the priorities of all groups having significant influence on the

\footnotetext{
${ }^{1}$ Now with the International Development Center of Japan, Tokyo, Japan.

Copyright $(\mathcal{C} 1982$ by the American Geophysical Union.
}

public decision-making process [Loucks et al., 1981, pp. 137-138]. Starr and Whipple [1980] discuss the differences in risk preferences exhibited by society and by individuals.

The multiobjective multiple-decision-maker character of public decisions is widely recognized, and multiobjective planning algorithms have been developed [Cohon, 1978]. The value of a multiobjective framework in water resources planning is that the benefit and disbenefit bundle associated with alternative projects and proposals can be better identified. As a result, the public as well as different participating public agencies and interest groups can better evaluate proposed projects using their own unarticulated objectives.

Advocated here is the inclusion of special risk-related system performance criteria within the multiobjective analysis of alternatives. By adding these performance measures to those already used to describe the expected costs and benefits of projects, individuals and groups should be better able to understand how a project might perform in the uncertain future. If they better understand how water resource systems may operate and how unpleasant any periods of unsatisfactory performance may be, individuals will be prepared to make better decisions.

Of interest are system performance criteria which are suitable for characterizing the stochastic and dynamic performance of such water resource systems as wastewater treatment plants, multireservoir water supply systems, or flood-flow forecasting and control systems. Some recent work on the properties of ecological systems is relevant to this problem.

Holling [1973] used the concept of resilience to describe the ability of a dynamic multispecies ecological system to persist with the same basic structure when subjected to stress. Resilience is to be contrasted with stability, which pertains to the variability of species densities over time. Holling points out that some systems may appear to be unstable because population densities vary over wide ranges. However, such systems may be very resilient, for they can persist after severe shocks or during periods of stress because of their capacity to accommodate variability in individual species densities. Very stable systems may not be able to cope with large variations in population densities. 
They may disintegrate if they suffer large losses due to fire or disease, the introduction of a new pollutant, or a radically new manageihent strategy.

Later work has extended this idea to environmental/ ecosystem management [Fiering and Holling, 1974; Holling, 1978]. These authors question the wisdom of management strategies which force natural systems to be highly stable. Enforcing stability may result in changes in the structure of managed systems which could greatly reduce their resilience. For example, enhancement of salmon spawning should lead to more productive fisheries and, as a result, greater fishing pressure. However, this greater pressure is very likely to cause the less productive stocks to become extinct or nearly so. This would leave the fishing ecosystem precariously dependent on a few artificially enhanced species [Larkin, 1979].

Several individuals have applied similar ideas to water and land related resource systems management. Haimes and Hall [1977] introduce several criteria for describing the characteristics of system models and planning situations. Fiering [1976, 1977] has developed measures of resilience which can be useful in water resource planning. Hashimoto [1980a,b] and Hashimoto et al., [this issue] have advanced the idea of system robustness, in which robustness describes the possible deviation between the actual costs of a proposed project and those of the least cost project design.

\section{Measures of System Performance}

In many studies the operational status of a water resource system can be described as either satisfactory or unsatisfactory. The occurrence of unsatisfactory performance will be described in this paper as a failure. A failure could correspond to the actual structural failure of a dam from a catastrophic flood event or an earthquake [Mark and StuartAlexander, 1977]. The modes of failure of concern here are less severe and more common. A failure may be a 50 -year or 200-year flood event which may cause extensive but not catastrophic flooding, moderate and severe droughts which make it impossible for reservoir systems to meet contractual obligations, or unexpected peaks in demand which tax water supply and wastewater treatment systems.

A number of indicators can be used to describe the possible performance of water resource systems. Simple and frequently used measures of system performance are the mean and variance of system outputs and performance indices. While the mean and variance of such quantities as project net benefits or DO concentrations in rivers are useful statistics, they are often not sufficient. In particular, the mean and variance describe the average level and average squared deviation from the mean of the parameters in question. These statistics provide a very vague description of just how poorly a system might behave in the infrequent situation when a failure does occur. The DO concentration in a river or the BOD removal rate in a wastewater treatment plant may be satisfactory 360 days a year. However, our primary concern may be the 5 days when things go wrong and aquatic communities might be seriously degraded (at least temporarily). For example, our attention should not be focused exclusively on the 10-year, 7-day low flow as things can be worse in critical parts of the river with the minimum 1-year, 7-day low flow due to the increased flow rates (Loucks et al. [1981], pp. 527-528, provide an example).

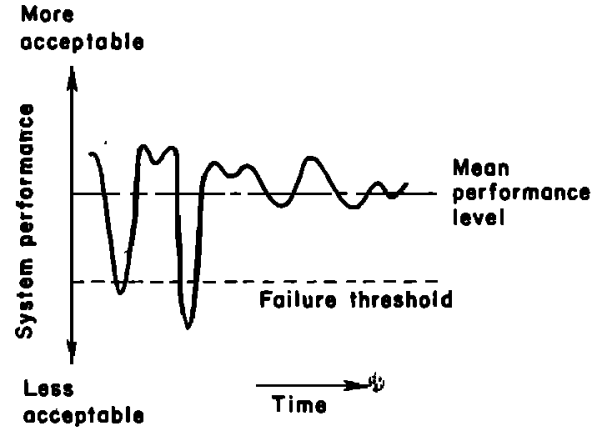

Fig. 1. Variable system performance with infrequent failures.

Figures 1 and 2 illustrate the inability of the mean and variance by themselves to define how severe and how frequent periods of poor performance may be. The figures contain a time history of the performance of two possible systems. The mean and variance of the performance parameter is the same in both cases over the time period shown. In fact, the curves are mirror images across their mean level. However, the performance history in Figure 1 displays two periods where performance clearly fell below the performance standard. This is never the case for the performance history in Figure 2.

When summarizing the values of performance parameters by their mean and variance, it is also difficult to determine if an improvement in the mean accompanied by an increase in the variance is an overall improvement. Theory addressing the relative tradeoff between the mean and variance of risky investments is well developed for small risks [Pratt, 1964]. However, if performance is highly variable or if the consequences of poor performance are severe, then it is appropriate and desirable to employ risk descriptors which (unlike the mean and variance of a parameter) describe in clear and meaningful terms what the character of failures might be.

Our analysis of system performance focuses on system failure, defined as any output value in violation of a performance threshold (such as a performance standard or a contractual obligation). System performance can be described from three different viewpoints: (1) how often the system fails (reliability), (2) how quickly the system returns to a satisfactory state once a failure has occurred (resiliency), and (3) how significant the likely consequences of failure may be (vulnerability). Descriptive as well as mathematical definitions of these criteria follow.

The definitions of these criteria are formulated assuming that the performance of the water resource system in ques-

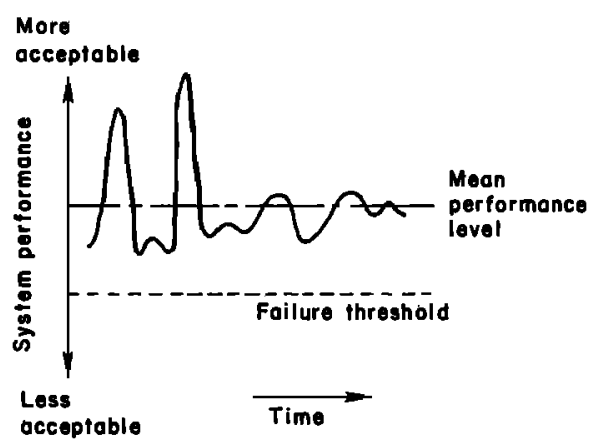

Fig. 2. Variable system peformance without failures. 
tion can be described by a stationary stochastic process. That is, the probability distributions that describe the output time series do not change with time. Of course this is only an approximation of reality but it is often quite reasonable. For instance, the probability distribution of streamflows at a particular site may change over time due to climatic shifts or land use changes in the drainage area. Still, it is both convenient and satisfactory in many cases to assume that streamflows are a stationary process over typical planning horizons.

\section{Reliability}

Denote a system's output state or status by the random variable $X_{t}$ at time $t$, where $t$ takes on discrete values $1,2,3$, - . . In general, the possible values of $X_{t}$ can be partitioned into two sets: $S$, the set of all satisfactory outputs, and $F$, the set of all unsatisfactory (failure) outputs. At any time $t$ the system output is assumed to be an element of one of these sets. The reliability of a system can be described by the frequency or probability $\alpha$ that a system is in a satisfactory state:

$$
\alpha=\operatorname{Prob}\left[X_{t} \in S\right]
$$

An alternate definition of reliability not adopted here is that reliability is the probability that no failure occurs within a fixed period of time, often taken to be the planning period. If the planning period is a single period, then the two definitions are equivalent.

Reliability is a widely used concept in water resources planning. Reliability is sometimes taken to be the opposite of risk. That is, the risk or probability of failure is simply one minus the reliability $\alpha$. Both reliability and this definition of risk do not describe the severity or likely consequences of a failure. The possible severity of failures can be described by other criteria, such as resiliency and vulnerability.

\section{Resiliency}

Resiliency will describe how quickly a system is likely to recover or bounce back from failure once failure has occurred. If failures are prolonged events and system recovery is slow, this may have serious implications for system design. One would like to design systems which can recover and return to a satisfactory state rapidly.

Resiliency may be given a mathematically precise definition. Let $T_{F}$ be the length of time a system's output remains unsatisfactory after a failure. The resiliency of a system can be defined as the inverse of the expected value of $T_{F}$. To derive a mathematical expression for that expected value, let

$$
\begin{array}{ll}
Z_{t}=1 & X_{t} \in S \\
Z_{t}=0 & X_{t} \in F
\end{array}
$$

Then $(1 / n) \sum_{t=1}{ }^{n} Z_{t}$ is the fraction of time from period $t=1$ to $t=n$ that the system output or performance is satisfactory. In the long run this fraction approaches the probability of the performance being satisfactory, and hence equals system reliability:

$$
\lim _{n \rightarrow \infty} \frac{1}{n} \sum_{t=1}^{n} Z_{t}=\alpha
$$

Let $W_{t}$ indicate a transition from a satisfactory to an unsatisfactory state:

$$
\begin{array}{lll}
W_{t}=1 & X_{t} \in S & X_{t+1} \in F \\
W_{t}=0 & \text { otherwise }
\end{array}
$$

In the long run the mean value of $W_{t}$ will equal the probability $\rho$ of the system being in the set $S$ in some period $t$ and going to the set $F$ in the following period:

$$
\rho=\operatorname{Prob}\left\{X_{t} \in S, X_{t+1} \in F\right\}=\lim _{n \rightarrow \infty} \frac{1}{n} \sum_{t=1}^{n} W_{t}
$$

The average sojourn time in the unsatisfactory or failure states during an $n$-period experiment is:

$$
\tilde{T}_{F}=\frac{A}{B}
$$

where $A$ is the total time in $F$ and $B$ is the number of times the process went into $F$. Hence

$$
\bar{T}_{F}=\frac{1}{n} \sum_{t=1}^{n}\left(1-Z_{t}\right)\left(\frac{1}{n} \sum_{t=1}^{n} W_{t}\right)^{-1}
$$

As $n$ approaches infinity, the average sojourn time $\bar{T}_{F}$ will approach its mean value $(1-\alpha) / \rho$. Thus the expected length of time that the system's output or performance remains unsatisfactory once it becomes unsatisfactory equals

$$
E\left[T_{F}\right]=\frac{1-\alpha}{\rho}
$$

This defines the average number of time periods a failure is expected to last once it has occurred. The inverse of this is the system's average recovery rate and is our measure of resiliency:

$$
\gamma=\frac{\rho}{1-\alpha}=\frac{\operatorname{Prob}\left\{X_{t} \in S \text { and } X_{t+1} \in F\right\}}{\operatorname{Prob}\left\{X_{t} \in F\right\}}
$$

In the long run, the number of transitions from satisfactory states in $S$ to unsatisfactory states in $F$ must equal the number of transitions in the reverse direction:

Prob $\left\{X_{t} \in S\right.$ and $\left.X_{t+1} \in F\right\}=$ Prob $\left\{X_{t} \in F\right.$ and $\left.X_{t+1} \in S\right\}$

Hence $\gamma$ is equivalent to the average probability of a recovery from the failure set in a single time step:

$$
\begin{aligned}
\gamma & =\frac{\operatorname{Prob}\left\{X_{t} \in F \text { and } X_{t+1} \in S\right\}}{\operatorname{Prob}\left\{X_{t} \in F\right\}} \\
& =\operatorname{Prob}\left\{X_{t+1} \in S \mid X_{t} \in F\right\}
\end{aligned}
$$

Note that if the occurrence of a failure $X_{t} \in F$ and a subsequent success $X_{t+1} \in S$ are probabilistically independent events, then $\gamma$ would reduce to Prob $\left\{X_{t+1} \in S\right\}$, which is our measure of reliability. 


\section{Vulnerability}

Here vulnerability refers to the likely magnitude of a failure, if one occurs. Even when the probability of failure is small, attention should be paid to the possible consequences of failure. Holling [1978] discusses the idea of safe-fail as opposed to fail-safe. Attempts to maximize system reliability are attempts to make a system's operation failure-free. Still, few systems can be made so large or so redundant that failures are impossible. Even when it is possible to raise levees high enough or make water supply reservoirs large enough that failure is hard to imagine, it is often not economical to do so. After a point, effort is better expended making the consequences of failure less severe and more acceptable than in trying to eliminate the possibility of failure altogether. Early warning systems, flood insurance, and flood-proofing of structures are three approaches to decreasing the costs of flooding when floods do occur. Likewise, the exclusion of buildings from floodways and the use of floodprone areas for parks, natural areas, and agriculture are other means of minimizing the costs of floods.

It is important to realize that efforts to maximize system efficiency and reliability can actually increase a system's vulnerability to costly failure should failure occur. Transformation of traditional agricultural systems to high yield single-species crops sets the stage for disaster should a new crop disease or pest develop. Likewise, flood control reservoirs and levees that control small floods create an image and sense of security; as a result, unwise development in partially protected areas can occur. This creates the potential for large losses should a large flood occur or a levee break. Replacement of small unreliable wastewater treatment plants by large well-managed regional facilities may decrease the frequency of plant failures, yet by concentrating the total treated wastewater flow in a single location, the impact and consequences of a breakdown in the biological oxidation process will be greatly magnified should the plant be overloaded or receive a slug of concentrated or toxic material [Adams and Gemmell, 1980].

The loss of a rear cargo door on the DC-10 aircraft due to improper latching provides an excellent illustration of failsafe versus safe-fail design. The blow out of the cargo door at high altitudes causes a rapid decompression of the cabin and the severing of control cables by the collapse of the floor separating the cabin and lower storage area. Commercial airlines emphasized design modifications and safety procedures to prevent such mishaps. Unfortunately, a failure occurred and many died. In some military aircraft, holes were cut in the floor separating the two compartments, allowing rapid decompression of the cabin should the cargo door be lost. This prevented structural damage to the aircraft and made the planes 'safe in failure.'

It is important that decision makers be aware of the vulnerability of a system to severe failure should a failure occur. This should be an important criterion in water resource system design and selection. To construct a mathematical index of system vulnerability, assume that the system performance variable $X_{t}$ can take discrete values $x_{1}$, $\cdots, x_{n}$. To construct a quantitative indicator of system vulnerability to severe failure should a failure occur, assign to each discrete failure state $x_{j} \in F$ a numerical indicator of the severity of that state, denoted $s_{j}$. Furthermore, let $e_{j}$ be the probability that $x_{j}$, corresponding to $s_{j}$, is the most
TABLE 1. Characteristics of River Flows

\begin{tabular}{lccc}
\hline & Winter & Summer & Annual \\
\hline Mean flows, $\times 10^{7} \mathrm{~m}^{3}$ & 4.0 & 2.5 & 6.5 \\
Standard deviation, $\times 10^{7} \mathrm{~m}^{3}$ & 1.5 & 1.0 & 2.3 \\
\hline
\end{tabular}

Correlation of flows: winter with following summer, 0.65 ; summer with following winter, 0.60 .

unsatisfactory and severe outcome that occurs in a sojourn into the set of unsatisfactory states $F$. Then $e_{j}$ equals Prob $\left\{x_{j}\right.$, corresponding to $s_{j}$, is the most severe outcome in a sojourn in $F$. One reasonable metric for overall system vulnerability would be the expected maximum severity of a sojourn into the set of unsatisfactory states:

$$
\nu=\sum_{j \in F} s_{j} e_{j}
$$

Here emphasis is placed not on how long failure persists (the inverse of resiliency) but on how bad things may become.

\section{REL!ABILITY, RESILIENCY, AND VULNERABILITY OF A WATER SUPPLY RESERVOIR}

Use of the reliability, resiliency, and vulnerability concepts is illustrated with a reservoir operation problem. For a reservoir of given capacity the reservoir operating policy determines the reliability, resiliency, and vulnerability of a water supply system. Kitson [1979] emphasized the need in reservoir operating policy development to consider reductions, during drought periods, in the amount of water available. He stated that this need leads to the concept of expressing reliability in terms of the frequency, duration and intensity with which restrictions have to be placed on water consumption.' Velikanov [1979], referring to irrigation water use, pointed to the necessity of evaluating in probabilistic terms system performance under conditions of both excessive and deficient water availability.

The reservoir operation example presented by Loucks et al. [1981, pp. 138-152] is used here to illustrate the use of risk-related system performance criteria. In that example a small reservoir with capacity $4 \times 10^{7} \mathrm{~m}^{3}$ was to provide 4.5 $\times 10^{7} \mathrm{~m}^{3}$ of water to meet summer irrigation needs. The logarithms of the inflows to the reservoir were modeled with a Thomas-Fiering model which reproduced the mean and variance of flows in each of two seasons and the season to

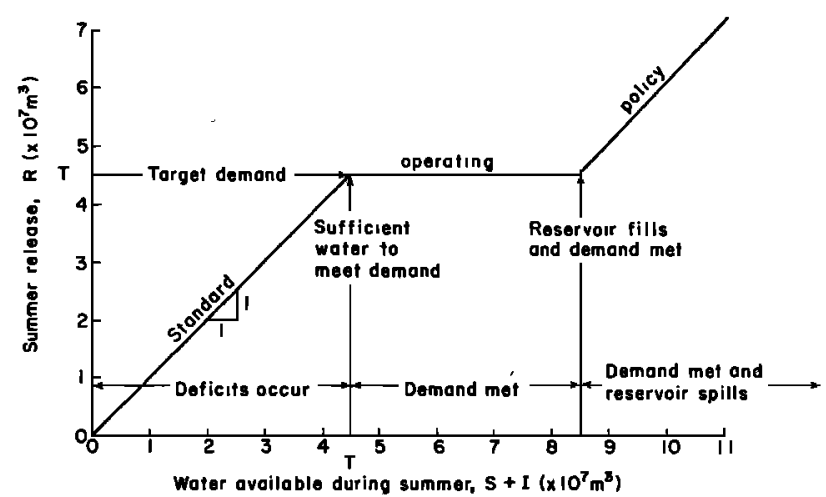

Fig. 3. Standard operating policy for initial storage $S$ and inflow $I$ obtained by minimizing the expected loss $E\left[l_{\beta}(R)\right]$ for $\beta=1$. 


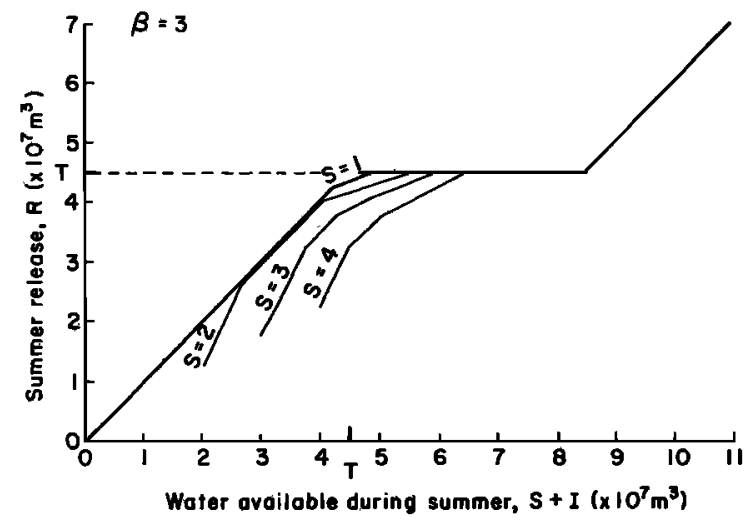

Fig. 4. Optimal summer release policy for $\beta=3$. The lines show best value of release $R$ as a function of initial storage $S$ plus inflow $I$ for specified value of $S$ and release target $T$.

season correlation of the flows (Loucks et al., 1981, pp. 141, 283-284, 305-307). The values of the statistics describing the relevant hydrology are given in Table 1 . It was also necessary to release $0.50 \times 10^{7} \mathrm{~m}^{3}$ of water during the wet season to satisfy minimum flow requirements.

The steady state operation of this simple system was simulated with a range of summer season operating policies. The winter operating policy was always to release $0.50 \times 10^{7}$ $\mathrm{m}^{3}$ of water if possible and to store as much of the excess water as the reservoir could hold. The summer season operating policies were derived by stochastic dynamic programing [e.g., Loucks et al., 1981, pp., 324-331] with the objective of minimizing the expected or average long-run loss:

$$
E\left[l_{\beta}(R)\right]
$$

where

$$
\begin{aligned}
T & \text { target release of } 4.5 \times 10^{7} \mathrm{~m}^{3} ; \\
R & \text { summer season release; } \\
l_{\beta}(R)=0, \text { when } R \geq T & \\
l_{\beta}(R)= & {[(T-R) / T]^{\beta}, \text { when } R<T . }
\end{aligned}
$$

The exponent $\beta$ defines the shape of the loss function $l_{\beta}(R)$. A range of $\beta$ values between 0 and 7 were considered to provide a range of policies. In the optimization, inflows, and storage volumes in each season were discretized in units of $0.25 \times 10^{7} \mathrm{~m}^{3}$. Optimal policies were a function of initial summer storage and the actual summer period inflow.

Note that the parameter $\beta$ is an artificial device introduced to facilitate the generation of operating policies which reflect different tradeoffs between shortfall magnitudes and failure frequency and hence different tradeoffs among reliability, resiliency, and vulnerability.

For $\beta=1$, one obtains the 'standard' operating policy shown in Figure 3. In the figure, $I$ denotes the summer inflow. The standard policy meets as much of the demand target as possible.

For $\beta>1$, operating policies exhibit 'hedging': they sometimes provide only a portion of the target release, when in fact all or at least more of the target volume could be provided. (Klemes [1977] and Stedinger [1978] discuss this phenomena.) This saves water to protect against future deficits which could be even larger. This is illustrated by the policy in Figures 4 and 5, obtained with $\beta=3$.

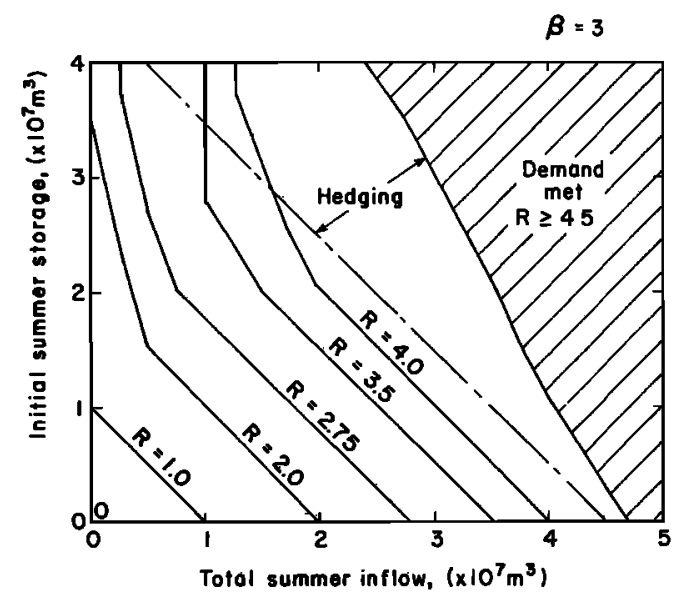

Fig. 5. Optimal reservoir summer release for $\beta=3$ as a function of initial storage and total summer inflow.

In Figure 4, several operating curves are discontinuous because they are defined over only a portion of the initial storage plus inflow $(S+I)$ axis. For example, if the initial summer storage is $S=3$, then the only legitimate values of $S$ $+I$ are those greater than or equal to 3 . As the two figures show, the optimal policy for $\beta=3$ can result in large and unnecessary deficits when the current summer inflow is below normal levels. To incur such deficits is optimal for the specified loss function, for it minimizes the expected value of immediate and possible future losses which could occur if streamflows remain below normal.

For $\beta<1$, a very different operating policy behavior results. In this case the marginal disutility of deficits is a decreasing function of the total deficit. As a result, optimal policies always meet the entire target if this is possible but sometimes fail to release any water at all when a modest failure is already unavoidable. Such a policy for $\beta=0.50$ is displayed in Figures 6 and 7.

In the limit as $\beta$ approaches zero, the loss function becomes

$$
\begin{array}{ll}
l_{0}(R)=0 & R \geq T \\
l_{0}(R)=1 & R<T
\end{array}
$$

In this instance the optimal policy is to meet the summer release target $T=4.5 \times 10^{7} \mathrm{~m}^{3}$ if possible and to deliver as

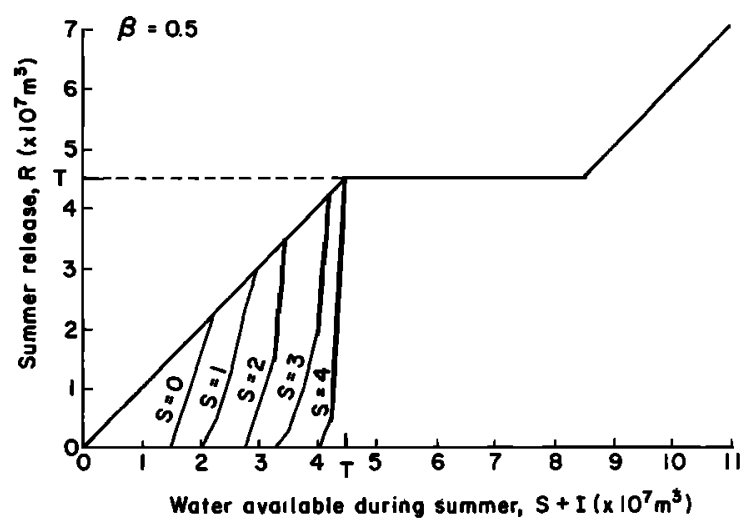

Fig. 6. Optimal summer release policy for $\beta=0.5$. The lines show best value of release $R$ as a function of available water $S+I$ for specified values of initial storage $S$. 


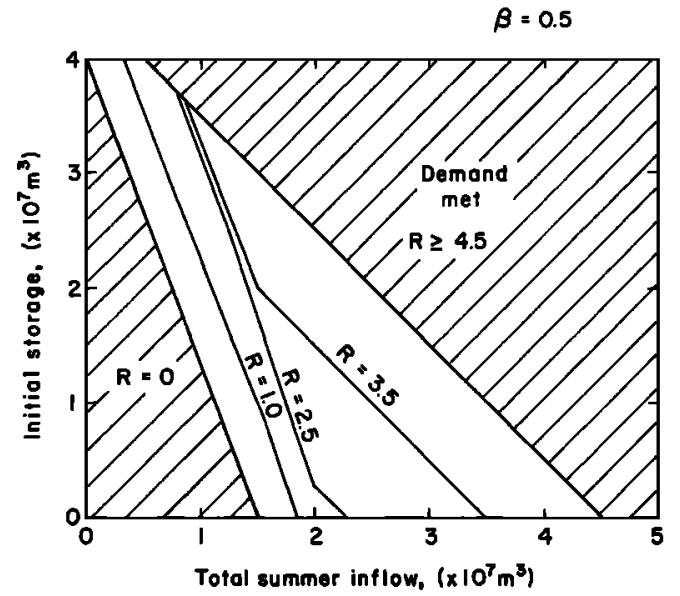

Fig. 7. Optimal reservoir summer release for $\beta=0.5$ as a function of initial storage and total summer inflow.

little water as one can if a failure cannot be avoided. This maximizes system reliability by saving water to avoid possible future failures when a failure in the current period is already unavoidable.

With each policy the reservoir-irrigation system was simulated for 10,000 years to determine (1) the reliability $\alpha$ with which the summer irrigation target was met, (2) the resiliency $\gamma$ of the system equal to the reciprocal of the average length of sequences of failure years, and (3) the vulnerablity $\nu$ of the system equal to the average of the maximum deficit that occurred in each sequence of failure years. A failure year occurred whenever the summer release $R$ was less than the target release $T$, equal to $4.5 \times 10^{7} \mathrm{~m}^{3}$.

Figure 8 illustrates the values of system reliability $\alpha$, resiliency $\gamma$, and vulnerability $\nu$ as a function of $\beta$, the exponent in the loss function used to derive the various operating policies. As $\beta$ increases, the penalty on large deficits becomes increasingly severe. As a result, as $\beta$ increases, system reliability $\alpha$ decreases because the optimal policies incorporate a propensity to incur small deficits so as to minimize the expected loss from larger deficits at later times.

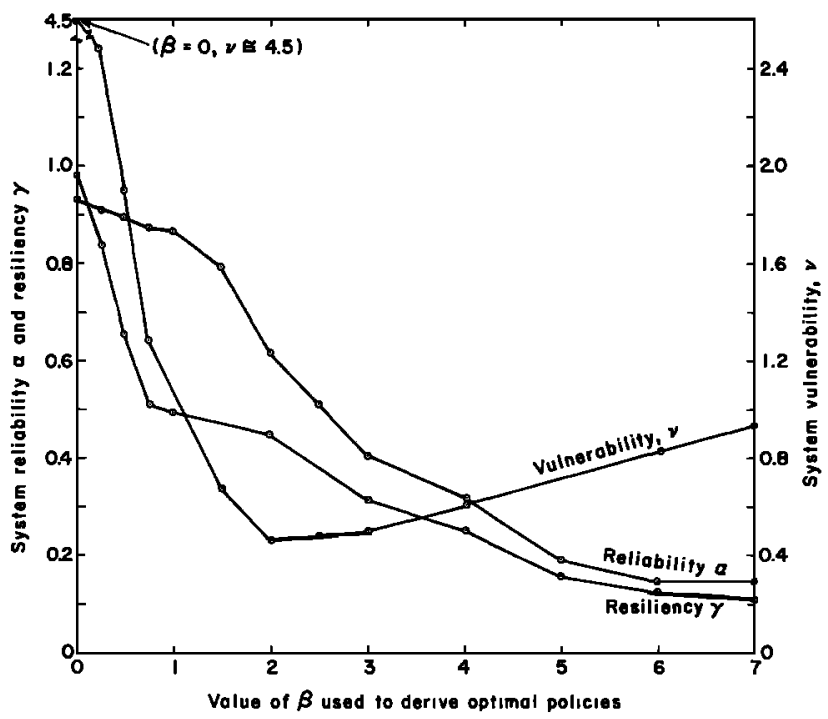

Fig. 8. System reliability, resiliency, and vulnerability as a function of parameter $\beta$ used to derive operating policies.
TABLE 2. Reliability and Expected Losses Achieved With Operating Policies Derived with Different Values of $\beta$.

\begin{tabular}{ccccc}
\hline $\begin{array}{c}\beta \text { Used } \\
\text { to Derive } \\
\text { Policy }\end{array}$ & $\begin{array}{c}\text { Reliability } \\
\text { of System } \\
\text { Operation, }\end{array}$ & \multicolumn{3}{c}{$\begin{array}{c}\text { Expected Value of Three Loss } \\
\text { Functions }\end{array}$} \\
\cline { 3 - 5 } & $\boldsymbol{0}$ & $E\left[l_{1}(R)\right]$ & $E\left[l_{2}(R)\right]$ & $E\left[l_{5}(R)\right]$ \\
\hline 0.25 & 0.93 & 6.6 & 6.5 & 6.1 \\
0.50 & 0.91 & 4.2 & 2.7 & 1.47 \\
0.75 & 0.89 & 3.2 & 2.0 & 0.98 \\
1.00 & 0.87 & 2.6 & 1.04 & 0.31 \\
1.50 & 0.87 & $2.5 *$ & 0.76 & 0.062 \\
2.00 & 0.62 & 2.6 & 0.70 & 0.051 \\
3.00 & 0.41 & 3.5 & $0.67^{*}$ & 0.040 \\
5.00 & 0.19 & 9.3 & 0.79 & 0.027 \\
7.00 & 0.15 & 12.4 & 1.37 & $0.022^{*}$ \\
& & & 2.2 & 0.029 \\
\hline
\end{tabular}

*Note that minimum value of $E\left[l_{k}(R)\right]$ is achieved at $\beta=k$ because the policy derived with given $\beta$ by construction minimizes $E\left[l_{\beta}(R)\right]$.

Resiliency generally shows the same trend as reliability. For $\boldsymbol{\beta}=\mathbf{0}$, system resiliency is high and sequences of failure years are very short. Deficits are very severe, often equaling the entire target. For $\beta \geq 3$, resiliency is low because periods of failure can be very long, although deficits are often small.

The vulnerability trend is different from that obtained with the other risk-related performance criteria. It achieves its maximum at $\beta=0$ when almost every failure is a complete failure. It then decreases with increasing $\beta$ to achieve a minimum at $\beta=2$. Above $\beta=2$, vulnerability actually increases with increasing $\beta$. This occurs because operating policies derived with large $\beta$ will frequently incur deficits much larger than is necessary. This saves water as a hedge against the possibility of even larger deficits in future periods. This tradeoff (for $\beta>2$ ) decreases the reliability and resiliency as well as the vulnerability of the system's performance. Still, it is optimal with respect to each policy's loss function. This is shown by Table 2, which reports the value of the expected loss function $E\left[l_{\beta}(R)\right]$ for $\beta=1,2$, and 5.

The values of reliability, resiliency, and vulnerability in Figure 8 reveal some of the characteristics of reservoir system performance that can be obtained with reservoir policies that minimize the specified loss functions. Realistic policies probably correspond to $\beta$ in the range of 1.0-2.0 and hence would have high reliability, modest resiliency, and close to minimal vulnerability. Figure 9 provides a more explicit description of the unavoidable tradeoff between vulnerability and reliability. One cannot have both the maximum possible reliability and minimum possible vulnerability.

\section{Conclusions}

In general, there exist tradeoffs among expected benefits, reliability, resiliency, and vulnerability. Use of the three risk criteria improves our ability to describe how often failures may occur, how long periods of unsatisfactory performance are likely to last, and just how severe failure might be. This was illustrated with a water supply reservoir example. There, high system reliability was accompanied by high system vulnerability. This information should be used to supplement other standard project evaluation criteria, including the distribution of project benefits and costs as well as various social and environmental impacts. By using 


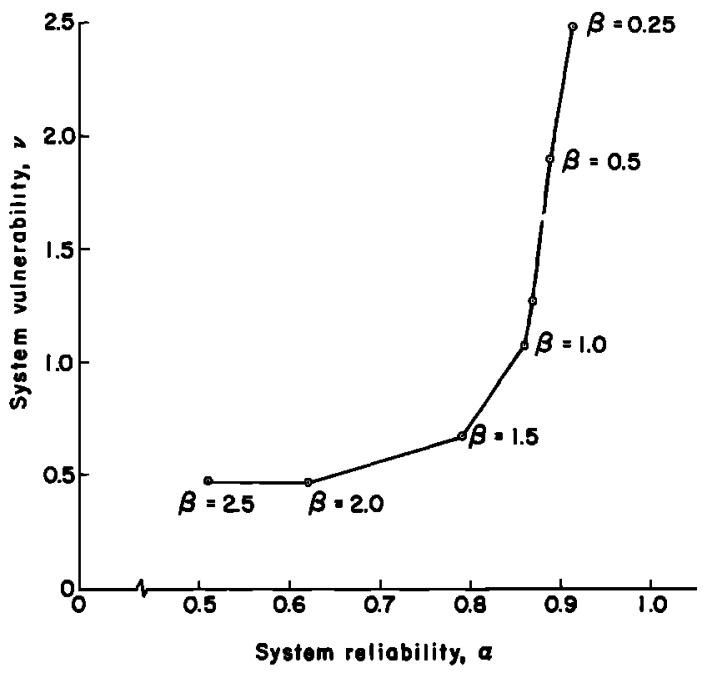

Fig. 9. Tradeoff between system reliability and vulnerability for $\beta$ between 0.25 and 2.5 .

improved descriptions of the possible nature of poor system performance, should it occur, individuals should be able to better understand the risks to which they are exposed by various project and no-project alternatives.

The particular mathematical definitions advanced here for resiliency and vulnerability should be viewed as illustrative examples. Every planning situation is in some way unique and calls for creativity in the definition of appropriate performance descriptors, such as resiliency, reliability, and vulnerability. It is unlikely that a single mathematical defintion of these concepts will be appropriate or useful in all situations. However, recognition and description of the possibility of low-probability but undesirable consequences of alternative plans should be an important component of the planning process. Hence engineers and planners need to develop appropriate quantitative risk criteria that describe the undesirable events that individuals may experience as a consequence of particular investment or operating policy decisions.

Acknowledgments. This research was supported in part by a grant from the Office of Water Research and Technology, U.S. Department of the Interior, and by the International Institute for Applied Systems Analysis, Laxenburg, Austria. We gratefully acknowledge the critical comments and suggestions of many, including those of M. Fiering, R. Schuler, and H. Taylor. R. Solanki's assistance with the numerical example is also appreciated. As always, the opinions expressed are not necessarily those of other individuals or organizations.

\section{REFERENCES}

Adams, B. J., and R. S. Gemmell, Water quality evaluation of regional wastewater management, J. Environ. Eng. Div., Am. Soc. Civ. Eng., 106(EE2), 437-450, 1980.

Benjamin, J. R., and C. A. Comell, Probability, Statistics and Decision for Civil Engineers, McGraw-Hill, New York, 1970.
Cohon, J. L., Multiobjective Programming and Planning, Academic, New York, 1978.

Davis, D. R., C. C. Kisiel, and L. Duckstein, Bayesian decision theory applied to design in hydrology, Water Resour. Res., $8(1)$, 33-41, 1972.

Fiering, M. B, The role of systems analysis in water program development, Nat. Resour. J., 16, 759-771, 1976.

Fiering, M. B, Preliminary notions on resilience, preliminary draft, Harvard Univ., Cambridge, Mass., April 1977.

Fiering, M. B, and C. S. Holling, Management and standards for perturbed ecosystems, Agro-Ecosystem, 1(4), 301-321, 1974.

Goicoechea, A., L. Duckstein, and M. Fogel, Multiple objectives under uncertainty: An illustrative application of PROTRADE, Water Resour. Res., 15(2), 230-210, 1979.

Haimes, Y. Y., and W. A. Hall, Sensitivity, responsivity, stability and irreversibility as multiple objectives in civil systems, Advan. Water Resour., I(2), 71-81, 1977.

Hashimoto, T., Robustness, reliability, resilience and vulnerability criteria for planning water resources systems, Ph.D. dissertation, Comell Univ., Ithaca, N. Y., 1980a.

Hashimoto, T., Robustiness criterion for planning water supply/ demand systems, Angew. Systemanal., 1(3), 137-144, $1980 b$.

Hashimoto, T., D. P. Loucks, and J. R. Stedinger, Robustness of water resource systems, Water Resour. Res., this issue.

Holling, C. S., Resilience and stability of ecological systems, Ann. Rev. Ecol. Systems, 4, 1-23, 1973.

Holling, C. S., Myths of ecological stability: Resilience and the problem of failure, in Studies on Crisis Management, edited by C. F. Smart and W. T. Stanbury, Butterworth, Montreal, 1978.

Keeney, R. L., and H. Raiffa, Decisions with Multiple Objectives: Preferences and Value Tradeoffs, John Wiley, New York, 1976.

Keeney, R. L., and E. F. Wood, An illustrative example of the use of multiattribute utility theory for water resources planning, Water Resour. Res., 13(4), 705, 1977.

Kitson, T., The operation of reservoir systems in Great Britain, overview paper presented at Workshop on Operation of MultiPurpose Multiple Reservoir Systems, Int. Inst. for Appl. Syst. Anal./IMGW, Jodlowy Dwor, Poland, May 28-June 1, 1979.

Klemeš, V., Value of information in reservoir optimization, Water Resour. Res., 13(5), 837-850, 1977.

Krzysztofowicz, R., and L. Duckstein, Preference criterion for flood control under uncertainty, Water Resour. Res., 15(3), 513$520,1979$.

Larkin, P. A., Maybe you can't get there from here: History of research in relation to management of Pacific Salmon, J. Fish. Res. Board Can., 36(1), 98-106, 1979.

Loucks, D. P., J. R. Stedinger, and D. A. Haith, Water Resource Systems Planning and Analysis, Prentice-Hall, Englewood Cliffs, New Jersey, 1981.

Mark, R. K., and D. E. Stuart-Alexander, Disaster as a necessary part of benefit-cost analyses, Science, 197, 1160, 1977.

Pratt, J. W., Risk aversion in the small and in the large, Econometrica, 32(1-2), 122-136, 1964.

Stedinger, J. R., Comment on 'Value of information in reservoir optimization,' Water Resour. Res., 14(5), 984-986, 1978.

Starr, C., and C. Whipple, Risks of risk decisions, Science, 208, 1114-1119, 1980.

Velikanov, A. K., The stochastic aspects of long-term water resources planning as applied to the integrated regional development problem, Collabor. Pap. CP-37-4, Int. Inst. for Appl. Systems Anal., Laxenburg, Austria, May 1979.

(Received June 6, 1980;

revised September 4, 1981;

accepted October 2, 1981.) 